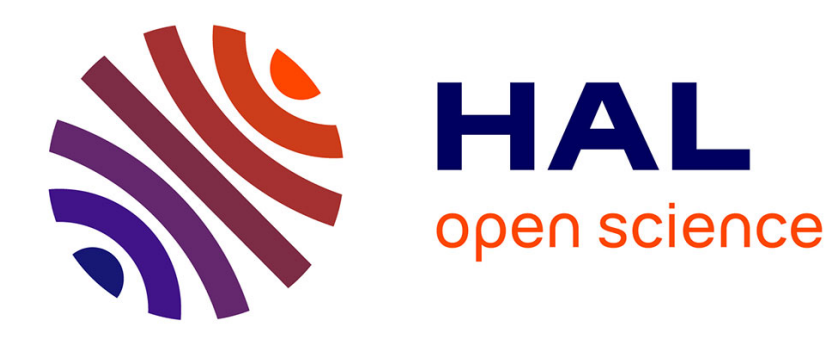

\title{
MICROWAVE EXPERIMENTS INCLUDING AVALANCHE
}

V. Dienys, A. Dargys

\section{To cite this version:}

V. Dienys, A. Dargys. MICROWAVE EXPERIMENTS INCLUDING AVALANCHE. Journal de Physique Colloques, 1981, 42 (C7), pp.C7-33-C7-49. 10.1051/jphyscol:1981704 . jpa-00221640

\section{HAL Id: jpa-00221640 https://hal.science/jpa-00221640}

Submitted on 1 Jan 1981

HAL is a multi-disciplinary open access archive for the deposit and dissemination of scientific research documents, whether they are published or not. The documents may come from teaching and research institutions in France or abroad, or from public or private research centers.
L'archive ouverte pluridisciplinaire HAL, est destinée au dépôt et à la diffusion de documents scientifiques de niveau recherche, publiés ou non, émanant des établissements d'enseignement et de recherche français ou étrangers, des laboratoires publics ou privés. 
V. Dienys and A. Dargys

Semiconductor physics Institute, Iithuanian SSR, Academy of Sciences, Vilnius, USSR

Résumé. - Dans cet exposé sommaire, on décrit les principes des techniques le plus souvent utilisées des micro-ondes destineés à I'étude des électrons chauas dans les semiconducteurs; on présente aussi l'état actuel des recherches dans le domaine des électrons chauds effectuées dans le champ électrique des micro-ondes. On souligne surtout les expériences ou l'on utilise la spécifité d'échauffement par le champ des micro-ondes aussi bien que celles qui ont permis de compléter l'information concernant le champ électrique continu.

Abstract. - The principles of the most popular microwave techniques for hot electron investigations in semiconductors are briefly described and the current status of hot electron investigations with microwaves is reviewed. The emphasis is put on the experiments which make use of the specific features of microwave heating or give an extra information in addition to that obtained from d.c. measurements.

1. Introduction. - The first microwave (mw) experiment on hot electrons was reported by Arthur et al. in 1956 / I/. It triggered a series of works devoted to the application of mw's for hot electron investigations. During a subsequent decade a lot of different $\mathrm{mw}$ techniques were proposed. At first they were intended for measuring the hot electron mobility /2-7/, but soon it was realized that mw experiments can give a valuable information on the hot carrier energy relaxation $18-11 /$.

The main subject of the report will be concerned with the hot electron studies during the last decade. During this period no essentially new ideas have appeared in the field of mw techniques. The known methods were further developed or have undergone some modifications and were applied to more refined physical problems. Therefore, at the begining of the paper the principles of the most popular Imw techniques will be briefly described. The rest of the paper will be devoted to the recent hot carrier experimental results obtained with mw's. Only the bulk properties of semiconductors will be concerned. 
2. Principles of $\mathrm{mw}$ techniques for hot electron investigation.

(i). Differential mobility technique. This technique was proposed by Arthur et al. /1/ and further developed by Gibson et al. 19/. A sample is mounted in a waveguide, Fig. la, which propagates a weak mw signal. Reflection $\Gamma$ and transmission $T$ coefficients are measured.

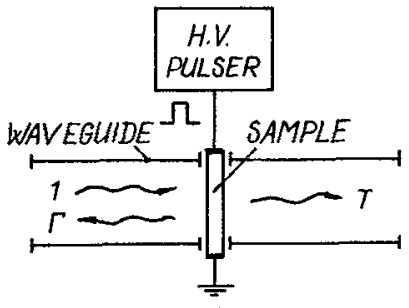

a)

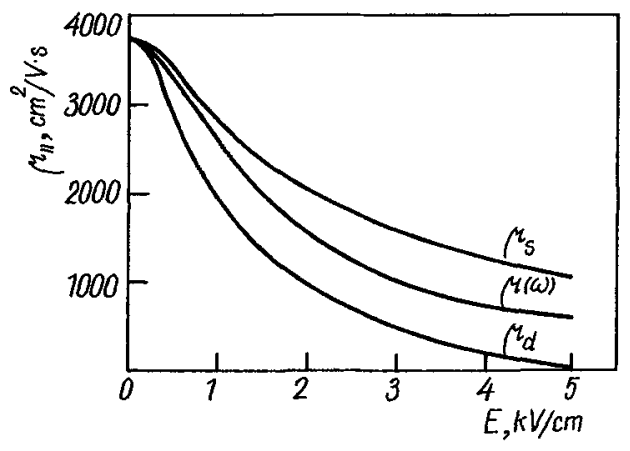

b)

Fig. I: a). The principle of the differential mobility technique. b). Experimental values of static $\mu_{s}=v_{d} / E$, differential $\mu_{d}=d v_{d} / d E$ and high frequency $M(\omega)$ mobilities as a function of dc electric field for n-type germanium, $\rho=4.68 \Omega \mathrm{cm}, T=300 \mathrm{~K}, \omega / 2 \pi=$ 34,75 GHz $/ 9 /$.

The application of strong pulsed dc electric field across the sample heats the carriers and as a consequence modulates $\Gamma$ and $T$ which can be used to find the small signal conductivity $\sigma(\omega)$ and the dielectric constant $\varepsilon(\omega)$ as a function of the dc electric field. Typical experimental results for the high frequency mobility $\mu_{\|}(\omega)=\sigma_{\|}(\omega) /$ en are shown in Fig. Ib in the case of parallel dc and mw fields. It is clear that in the zero frequency limit $\mu_{\|}(\omega-0)$ corresponds to the aifferential mobility $\mu_{d}=d v_{d} / d E$, where $v_{d}$ is the drift velocity and $E$ is the electric field intensity. Inspection of Fig. Ib shows that $\mu_{11}(\omega)$ exceeds $\mu_{d}$. The observed difference can be explained by the delay effects due to the finite rate of energy relaxation.

(ii). Integral mobility technique. With the sample mounted similarly, the carriers are heated by the strong mw electric field $E$, cos $\omega t$ while sensing is made by the weak dc field $E_{0}$, Fig. 2a. The measured quantity is an average current $\langle j\rangle$ flowing through the sample

$$
\langle j\rangle=\frac{1}{T} \int_{0}^{T} j\left(E_{0}+E_{1} \cos \omega t\right) d t,
$$

where $T=2 \pi / \omega$. A typical dependence of $\langle j\rangle$ on $E_{1}$ is illustrated in Fig. 2b. To obtain the current-field characteristic from the observed $\langle j\rangle$ vs $E_{1} E q .(I)$ must be inverted. This is possible to do only 


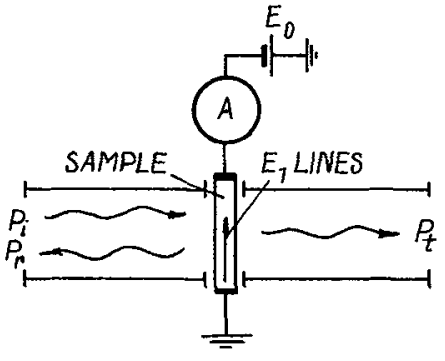

a)

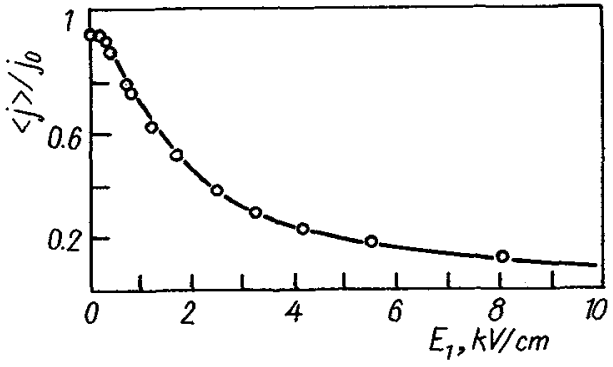

b)

Fig. 2: a). The principle of the integral mobility technique. $P_{i}, P_{r}$ and $P_{t}$ are the incident, reflected and transmitted power, respectively.

b). Ratio of average currents with, $\langle j\rangle$, and without, $j_{0}$, excitation vs peak value of the Inw field for n-type germanium, $\rho=$

$5 \Omega \mathrm{cm}, \quad T=300 \mathrm{~K}, \omega / 2 \pi=2,85 \mathrm{GHz} / 6 /$.

in the low frequency limit when the relaxation effects are negligible, for example, by numerical techniques or, as a common practice is, by resorting to schlomilch's integral equation.

The main difficulty of this technique is to determine $E_{1}$ inside the sample. Depending on the sample impedance $Z_{s}$ two different approaches are usually used. If $Z_{s}$ is of the order of a waveguide impedance $Z_{g}$ the value of $E_{1}$ is deduced from the amount of the absorbed mw power by the sample/4/. On the other hand, if $Z_{s} \gg Z_{g}$ the mw field in the sample is assumed to be uniform and equal to that in the empty guide /6/.

(iii). Harmonic mixing technique. As far back as 1949 it was shown that ractification can occur in a symmetric nonlinear element if applied signal consists of several sinusoids with frequencies in a cer tain ratio/12/. Experimentally this was verified with magnetic circuits containing ferromagnetic material. A similar effect was observed in hot electron mw experiment by Carlin and Porhela/13/in 1965, and a year later Schweitzer and Seeger proposed to use it for the measurement of energy relaxation time $\tau_{\varepsilon} / 14 /$. In the simplest case of homogeneous sample, having the static current density-field characteristic

$$
j=\sigma_{0}\left(1+\beta E^{2}\right) E
$$

the electric field

$$
E=E_{1} \cos (\omega t+\varphi)+E_{2} \cos 2 \omega t
$$


produces a dc open-circuit field

$$
E_{\text {mix }}=\frac{3}{4} \beta\left(7+4 \omega^{2} \tau_{\varepsilon}^{2}\right)^{-1 / 2} E_{1}^{2} E_{2} \cos 2(\varphi+\psi)
$$

Here $\sigma_{0}$ is the zero-field conductivity and $\beta$ is the warm electron coefficient. Because of the energy relaxation an aditional phase shift $\psi$ appears which is related to $\tau_{\varepsilon}$ by

$$
\operatorname{tg} 2 \psi=2 \omega^{3} \tau_{\varepsilon}^{3} /\left(1+3 \omega^{2} \tau_{\varepsilon}^{2}\right)
$$

The schematic diagram of an experimental setup is given in Fig. 3a. To find $\tau_{\varepsilon}$ it is most convenient to measure the phase shift $\psi$

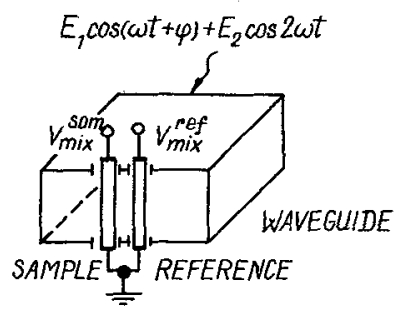

a)

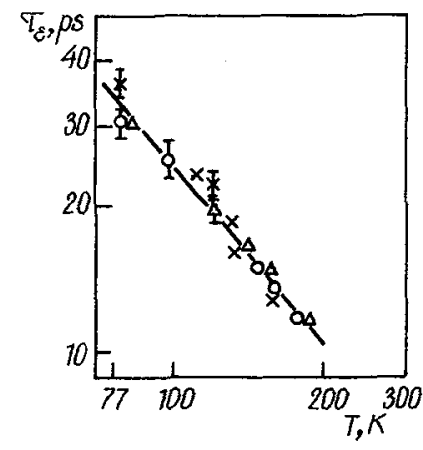

b)

Fig. 3: a). Sample holder for harmonic mixing experiments.

b). Energy relaxation time vs lattice temperature in n-type germanium, $0-5 \Omega \mathrm{cm}, \Delta-12 \Omega \mathrm{cm}, x-30 \Omega \mathrm{cm} / 15 /$.

with respect to the reference sample in which the condition $\omega \tau_{c} \ll 7$ is satisfied or $T_{\varepsilon}$ is known beforehand, and then to use (5). Fig. $3 b$ illustrates the dependence of $\tau_{\varepsilon}$ on lattice temperature obtained in this way.

(iv). Harmonic generation technique. First experiments on the harmonic generation by hot electrons were concerned with the problem of constructing an efficient frequency multiplier /16,17/. However, the conversion efficiency of such multipliers has been found to be rather low, especially, for high frequencies where the inertia of carrier heating becomes the main limiting factor /18/. As a result the frequency multiplication has not found a widespread application in $\mathrm{mw}$ engineering. On the other hand, in semiconductor physics it was successfully applied for hot electron energy relaxation investigation /18-20/. Fig. 4a illustrates the idea of the experiment. A rodshaped semiconductor sample is mounted in the middle of the window of a rectangular waveguide. If $\mathrm{mw}$ and ac fields are impressed in the sample simultaneously it irradiates the higher harmonics which could 


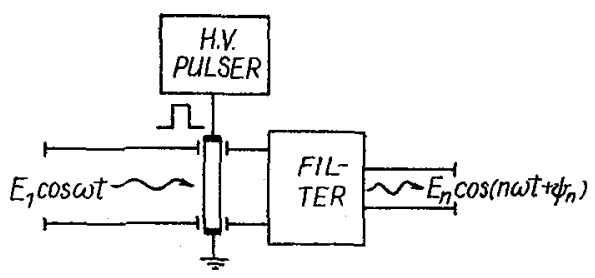

a)

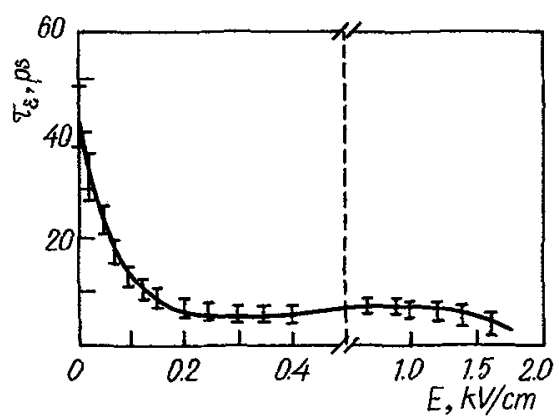

b)

Fig. 4: a). The principle of the hamonic generation technique.

b). Energy relaxation time vs electric field for n-type germanium at $77 \mathrm{~K}, \rho=40 \Omega \mathrm{cm} / 20 /$.

be separated out, for example, by the guide tapered to the smaller dimensions or by a harmonic filter. The amplitude and phase of the harmonic of interest depend on the carrier heating inertia, and this property may be used to obtain the energy relaxation time. For example, the harmonic generation technique was used for the investigation of the dependence of $\tau_{\mathcal{E}}$ on electric field in $n-G e$ and $n-S i / 20 /$. Some results of that paper axe plotted in Fig. $4 \mathrm{~b}$.

(v). Gyclotron resonance technique. The cyclotron resonance experiments on semiconductors are usually employed to study their band structure. In addition the width of the resonance line contains information on the collision frequency of carriers with lattice imperfections. This property can be used to study the carrier heating in the cyclotron resonance experiments by measuring the dependence of the line shape on the absorbed mw power, Fig. 5.

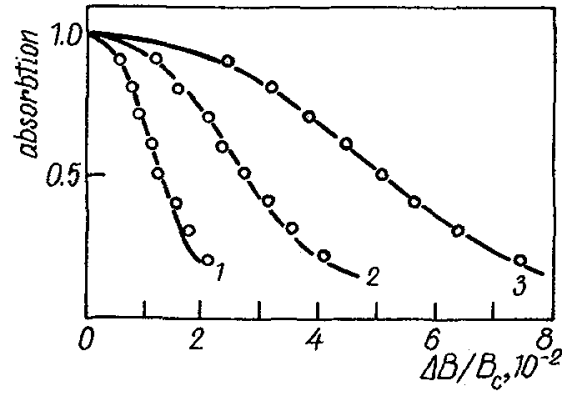

Fig. 5: Cyclotron line shapes for various power levels 121/. The absorbed mw power increases with the number of curves. 
The described mw techniques, in some sense, may be considered as fundamental ones. A few words should be mentioned of other related techniques. In /22/ the method employing a cavity with the sample partially replacing the cavity walls was used to measure the hot carrier small signal complex conductivity. In /23/ it was shown that the observation of the complex conductivity at the fundamental frequency of high mw signal may be used to find the velocity-field characteristic. In /24/ a hybrid of mw and conventional time-of-flight techniques was proposed to measure drift velocity in thin low resistivity samples, and etc.

3. Relaxation effects. - High electric field causes a departure of the energy and momentum distribution of carriers from equilibrium state. Usually the response times characterizing the energy and momentum loss to the lattice are in the range from $10^{-7} \mathrm{~s}$ to $10^{-13} \mathrm{~s}$. Thus the frequency is high enough for strong relaxation effects to appear, and direct observation of the inertia of physical processes under hot electron conditions is possible. This is of considerable interest for semiconductor physics. The progress in the relaxation effect study during the last decade was achieved mainly in three aspects. First, apart from the traditional materials Ge and Si also other materials, especially the group $A_{3} B_{5}$ semiconductors, have been extensively studied, see e.g. /25-28/. Second, the experiments have become more sophisticated and comprehensive. As a result the

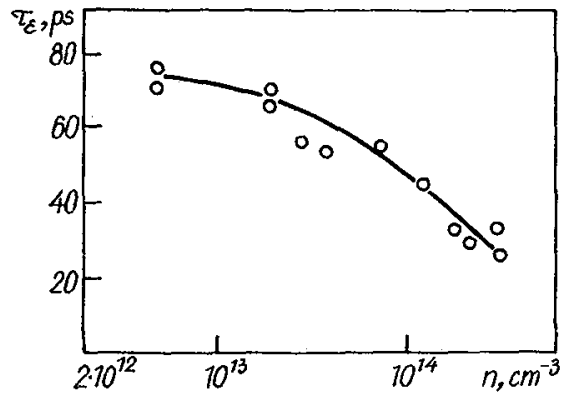

a)

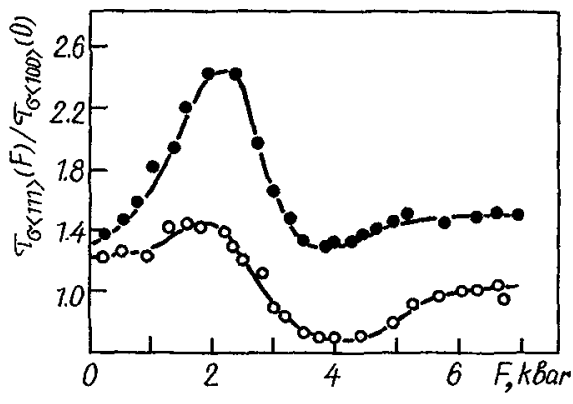

b)

Fig. 6: a). Fnergy relaxation time vs carrier concentration for $n$-type germanium at $77 \mathrm{~K} / 29 /$.

b). The dependence of conductivity relaxation time $\tau_{\sigma}$ measured by the harmonic mixing technique on the uniaxial compressive stress $F$ in n-type germanium. $T=80 \mathrm{~K}, \vec{E} \| \vec{F} /\langle\langle 71\rangle, \omega / 2 \pi=9.41 \mathrm{GHz}, \cdot-n=$ $=2.4 \times 1014 \mathrm{~cm}^{-3}, 0-n=5 \times 1012 \mathrm{~cm}^{-3} / 32 \%$.

dependencies of the energy dissipation rate on the electric field 
intensity /20,25,27/, free carrier concentration /29/, uniaxial strees /30-33/ have been measured. For illustration Figs. 4b, 6 and 7 show some of the results. Valuable information on the carrier sca-

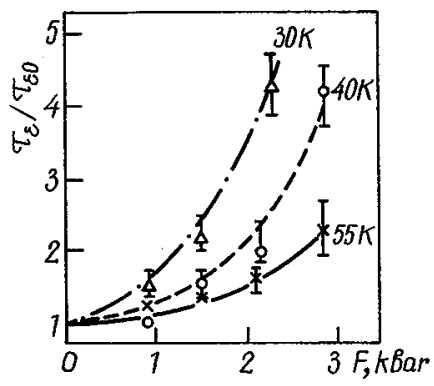

Fig. 7: Energy relaxation time vs uniaxial stress in ptype germanium at different temperatures. Stress applied in the $\langle 100\rangle$ direction, $T_{\varepsilon 0}$ denotes the energy relaxation time at zero stress $/ 33 /$.

ttering mechanisms may be deduced from these and similar data. For example, from the results displayed on Fig. 6 it can be concluded that in $\mathrm{n}-\mathrm{Ge}$ the strengths of the intervalley electron-electron (e-e) scattering and the intravalley e-e scattering are of the same order.

Finally, the investigations of relaxation effects in the wide frequency range was recently undertaken /34-36/. In contrast to the single frequency relaxation effect measurements such investigations can yield more information, especially on the importance of various relaxation channels. We shall dwell on these experiments in more detail. Till now they have been performed in the warm electron region where with the help of Eq. (I) one can show that

$$
<j\rangle=\sigma_{0} E_{0}\left(1+\frac{1}{2} \beta^{*} E_{1}^{2}\right) \text {. }
$$

Here $\beta^{*}$ denotes the frequency dependent effective warm electron coefficient. In principle the analysis of $\beta^{*}$ as a function of the frequency allows to find the energy relaxation time / $8 /$. However as is seen from Eq. (6) the amplitude of the electric field $E_{1}$ in the sample must be known what is rather difficult to do, in a wide frequency range. For this reason it is advantageous to redefine Eq. (6) through quantities which can be measured directly, namely:

$$
\langle j\rangle=\sigma_{0} E_{0}\left(1+\alpha^{*} \frac{P}{\sigma_{0}}\right),
$$

where $P$ is the mw power absorbed in a unit volume of the sample. 
Eq. (7) may be considered as a new definition of the effective warm electron coefficient $\alpha^{*}$. This modification allows to simplify the experimental procedure essentially. The absorbed power $P$ can be measured by the conventional bridge circuit method /37/ in which the sample under investigation simultaneously serves as a bolometer. The mw signal is square-wave modulated, the modulation frequency beeing sufficiently high to suppress the ac component of lattice heating.

To illustrate the possibilities of the method the dependence of $\alpha^{*}$ on the frequency for $n-S i$ and $p-G e$ is shown in Fig. 8. In case of n-Si, Fig. 8a, the experimental results fit well the phenomenological relation

$$
\alpha^{*}(\omega)=\alpha^{*}(0) \frac{1}{3}\left[1+\frac{2+\omega^{2} \tau_{\varepsilon} \tau_{m}}{1+\omega^{2} \tau_{\varepsilon}^{2}}\right],
$$

where $\tau_{m}$ is the momentum relaxation time. For $\mathrm{p}-\mathrm{Ge}$ the dependence

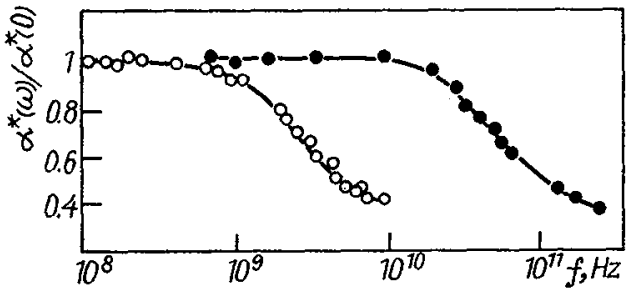

a)

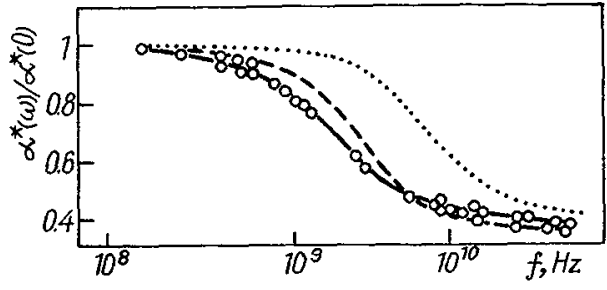

b)

Fig. 8: The frequency dependence of the coefficient $\alpha^{*}$

a). $n-\mathrm{Si}, \quad n=2 \times 10^{13} \mathrm{~cm}^{-3}, E \|\langle 771\rangle, \circ-T=80 \mathrm{~K}, \cdot-T=290 \mathrm{~K}$. Solid curves represent the best fit of Eq. (8) with the experimental

b). $\mathrm{p}-\mathrm{Ge}, \quad p=5.4 \times 10^{13} \mathrm{~cm}^{-3}, \vec{E} \mid K\langle 700\rangle, \quad T=80 \mathrm{~K}$. Points are experimental data. The dashed line represents the best fit of Eq. (8) with the experimental data ( $\left.\tau_{\xi}=90 \mathrm{ps}, \tau_{m}=5.5 \mathrm{ps}\right)$. The dotted Iine shows the dependence predicted by the Eq. (8) for $\tau_{\varepsilon}=30 \mathrm{ps}$ 115/. The solid curve represents the best fit of the experimental data with the phenomenological expression corrected for the interband repopulation of holes ( $\tau_{\varepsilon}=110 \mathrm{ps}, \tau_{m}=5.5 \mathrm{ps}$, interband repopulation time $\left.\tau_{i}=8 \mathrm{ps}\right) / 36 /$.

is more complicated, Fig. 8b. The analysis of experimental data of $\mathrm{p}-\mathrm{Ge}$ have shown that in this case the conductivity relaxation is determined not only by the energy dissipation process but also by the hole interband redistribution as well as by the accumulation of holes 
in the field direction /36/.

4. Impact ionization. - In high electric fields the avalanche breakdown sets in with the consequence that carrier concentration changes with the electric field. In mw experiments due to the breakdown of air the electric field intensity in a sample usually does not exceed $\sim 3 \cdot 10^{4} \mathrm{~V} / \mathrm{cm}$. Such fields are too small to initiate the band-band impact ionization in many traditional semiconductors. Therefore, the mw induced carrier multiplication was observed mainly as a result of impurity breakdown at low temperatures /38/, or in narrow-gap semiconductors, see e.8./39-44/. In first experiments the onset of breakdown was deduced from the transmitted mower level through a thin semiconductor plate completely filling the waveguide cross-section. Typical experimental results are shown in Fig. 9. The break of

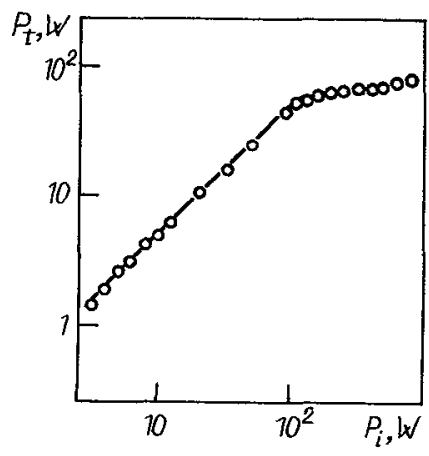

Fig. 9: Now transmission through InSb plate at $80 \mathrm{~K}$ $139 /$.

the curve is identified with the onset of the carrier multiplication. However, such measurements are used seldom since only a very crude estimation of the electric field intensity in the plate is possible.

The integral mobility technique has been found as a more suitable method for the investigation of avalanche properties in semiconductors due to the following reasons. First, the electric field in the rod-shaped sample can be made uniform and found from the absorbed $\left(Z_{s} \sim Z_{g}\right)$ or the transmitted $\left(Z_{s}>Z_{g}\right)$ power. Also, the identification of the breakdown is easier. Fig. IO shows the dependence of the threshold field of impact ionization $E_{T}$ on the lattice temperature for p-InSb at $10 \mathrm{GHz}$ and $37 \mathrm{GHz}$ as found by this method. The difference in $E_{T}$ vs frequency is a manifestation of the delay of the impact ionization process. 


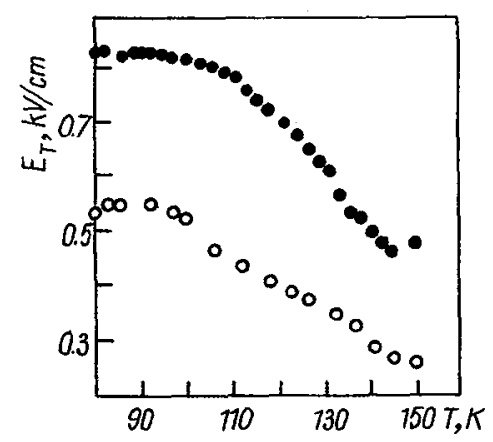

Fig. 10: Threshold field of inpact ionization vs lattice temperature in $\mathrm{p}$-InSb at different mw frequencies. $\circ-10 \mathrm{GHz},-37 \mathrm{GHz} / 42 /$.

Recently it was demonstrated that mw technique can be successfully employed for the study of the impact ionization and magnetotransport in crossed electric and magnetic fields /41,43,44/. At mw frequencies the electric field is applied contactlessly, therefore, a premature breakdown as observed in dc electric and magnetic fields due to shorting of the Hall field by current contacts can be avoided. Also,because of a finite charge redistribution time it is possible to control the magnitude of the $\mathrm{mw}$ Hall field in the samples with the Hall geometry. For example, by choosing small enough carrier concentration or by placing the sample in a high magnetic field $B$ $(\mu B \gg 1)$ one can reduce the nw Hall field nearly to zero. In Fig. 11

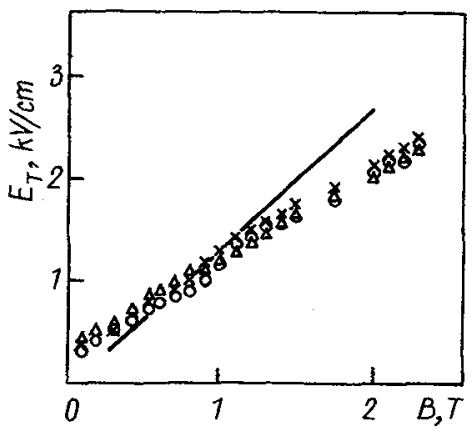

Fig. 11: Threshold field of impact ionization vs magnetic field for $n$-Insb samples with resistivities at $77 \mathrm{~K}, 0.17 \Omega \mathrm{cm}(0), 22 \Omega \mathrm{cm}$ $(x), 54 \Omega \mathrm{cm}$ (4) /43/. The solid line shows the prediction of theory / $45 /$ assuming the impact ionizing collision time $\tau_{i c}=10$ ps.

$E_{T}$ vs $B$ is shown for n-InSb at $80 \mathrm{~K}$. At the frequency of measurement, 9.4 GHz, the mw Hall field was negligible and samples behaved as if being of the Corbino geometry. As expected in the high magnetic field region $E_{T}$ increases nearly linearly with $B$. 
The nonlinear mw magnetoplasma resonance in small samples was demonstrated to be useful to study the impact ionization /44/. With samples in the form of small discs placed in a rectangular guide, a dependence similar to that presented in Fig. Il was obtained.

The avalanche breakdown is associated with the pair production scattering which may be described by introducing the ionizing collision time $\tau_{i c}$. If ionizing collisions predominate and $\omega T_{i c} \geqslant 1$ one expects the relaxation effects due to the avalanche to be observable in the transport coefficients. For example, the ionizing collisions are expected to reduce or even suppress the negative differential mobility $/ 46 /$. In search of this effect the drift velocity in $n$-InSb was measured deep into the avalanche region /47/. To factorize the current into the drift velocity $v_{d}$ and carrier concentration $n$ the double mw pulse was used. If separation between the pulses $\Delta t$ is smaller than the pair lifetime the nonequilibrium carrier concentration will not change appreciably during this time. Therefore $n$ can be deduced from the conductivity measured during the interval between pulses when the excess carriers are in the thermal equilibrium with the lattice. As seen from Fig. 12 the carrier concentration

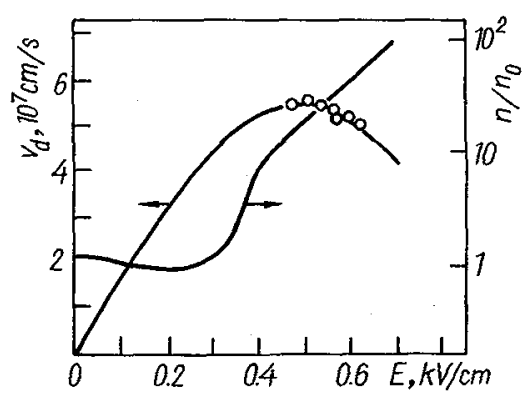

Fig. 12: The dependence of arift velocity and nonequi librium carrier concentration on electric field in n-InSb obtained with double pulse mw technique /47/. The dots are experimental results obtained by the time-of-flight method $/ 48 /$.

steeply increases at fields higher than $250 \mathrm{~V} / \mathrm{cm}$. The drift velocity reaches the maximum value at $500 \mathrm{~V} / \mathrm{cm}$ and then drops down. No transition from the negative to the positive differential mobility is observed as predicted by the Monte Carlo calculations /46/ if ionization frequency is assumed to be larger than phonon scattering frequency.

5. Negative differential mobility (ndm). - In connection with transferred electron devices the measurement of velocity-field ( $v-E$ ) characteristics in materials with ndm is of fundamental importance. The 
integral mobility technique has been widely applied in the investigations of GaAs and related compounds to supplement the results obtained with other techniques. At present practically it is the only method to obtaine a v-E curve possesing ndm in high electron density semiconductors because the most direct method, the time-of-flight method, unfortunately needs low carrier density material. A review of typical experimental results and problems connected with mw measurements in the ndm region may be found, e.g. in/49/. Therefore onIf two experiments which illustrate the specific possibilities of $\mathrm{mw}$ technique will be mentioned.

Most of the investigators dealing with the ndm have been interested in finding $\mathrm{V}-\mathrm{E}$ characteristic. On the other hand, the dependence of the small signal complex conductivity on dc heating field

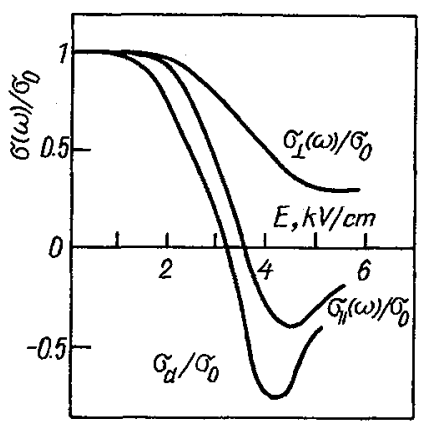

a)

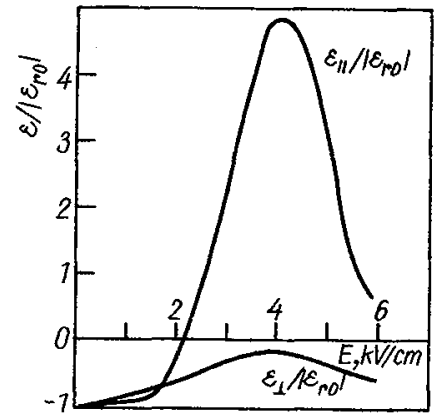

b)

Fig. 13: Small signal conductivity (a) and dielectric constant (b) vs heating field for the cases of parallel (II) and perpendicular ( $\perp$ ) $50 \mathrm{GHz}$ sensing field in n-GaAs. The curves are normalized to the zero heating field values. $T=300 \mathrm{~K}, \rho=21 \Omega \mathrm{cm} / 50 /$.

in the ndm region is of a considerable interest. It has been demonstrated that the $\mathrm{mw}$ technique is suitable in such situations /50/. To suppress the high field domain formation the bias high electric field has been created by applying $10 \mathrm{GHz}$ m's. A $50 \mathrm{GHz}$ weak sensing field was applied simultaneously to find the complex small signal conductivity as a function of the bias field. Fig. 13 shows the real and imaginary parts of the small signal conductivity of $n-G a A s$ at $300 \mathrm{~K}$ for parallel and perpendicular directions of the heating field vector with respect to the sensing one. The obtained results are valuable in understanding hot electron dynamics.

In / $51 /$ the investigation of ndm in the graded gap mixed crystals $A x_{x}$ Ga $_{1-x^{A s}}(x=0-0.17)$ were performed. The results give an evidence that in graded gap materials the lateral transport can be im- 
portant. The problem of the lateral transport will be treated in detail in the paper by Hess at this conference.

6. Nonuniform structures. - The mw technique allows effective decoupling of the large heating and small de measured fields. Thus the difficulties encountered by first investigators in thermoelectric power studies by dc fields have been overcome by mw's /52,53/. Nevertheless, the interpretation of thermoemf arising between semiconductor and metal due to mw field was difficult, mainly, because the electric field distribution near the contact was strongly inhomogeneous and the contribution of contact was found to depend on the nature of the transition region between the semiconductor and the metal. Various spurious voltages were difficult to eliminate as well. Later it was proposed to use high resistivity samples with graded $n^{+}-n$ (or $p^{+}-p$ ) junction /54/. To eliminate field distortions due to conduction electrons the junction length and resistivy must meet the conditions $L \gg \omega / v_{d}$ and $\omega T_{M} \gg 1$, where $\tau_{M}$ is the Maxwellian relaxation time. With uniform mw field distribution in the junction the interpretation of the experimental results is much simpler. In particular, it can be shown that under the action of mw field over the junction a hot carrier emf $V_{T}$ appears which can be used to deduce the hot carrier diffusion coefficient. With the mw field parallel and perpendicular to the concentration gradient the technique allows to find the longitudinal or transverse hot carrier diffusion coefficient.

A schematic arrangement for the longitudinal thermopower measurement is represented in Fig. 14a, while Fig. 14 b shows the longitudinal diffusion coefficient in $\mathrm{n}-\mathrm{Si}$ at $300 \mathrm{~K}$ obtained with lithium diffused $n^{+}-n$ junction. Later the same technique was extended to Schottky barriers fabricated on high resistivity semiconductors /55/.

7. Interaction between different groups of carriers. - In semiconductors with different groups of carriers, e.g. many-valley, intrinsic, degenerate-band semiconductors, the amount of energy extracted from the field and delivered to the lattice is governed both by the interaction of carriers with the lattice and by the interaction between groups. The latter has been observed in the hot carrier cyclotron resonance experiments in p-Ge through the dependence of linewidth on the mower /57,58/. A more direct observation of the interaction between groups would be possible if selective heating and 


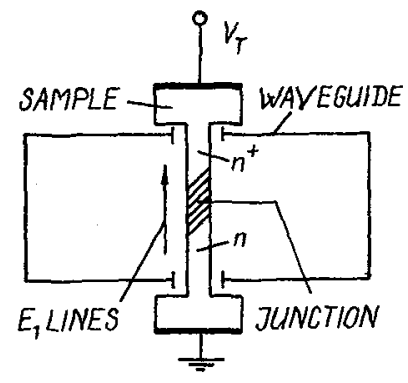

a)

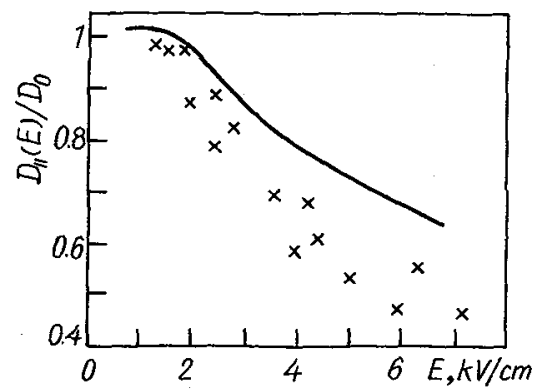

b)

Fig. I4: a). The principle of the mw technique for the longitudinal diffusion coefficient measurement. Typical junction lengths are from 0.1 to $2 \mathrm{~mm}$ and concentration ratio $\mathrm{n}^{+} / \mathrm{n}$ is from 2 to 4 . b). Hot electron longitudinal diffusion coefficient vs $E$ in a high resistivity $n-S i$ at $300 \mathrm{~K}$. The solid line represents the values of $D_{l l}$ obtained by mw's $155 /$ and crosses are measured by the time-of-flight technique $156 /$. The data are normalized to the zero field value of diffusion coefficient $D_{0}$.

sensing of the various groups of carriers were performed at different frequencies. Such cross-modulation type experiments were demonstrated in /59/. The modulation at the sensing frequency was caused by the change of carrier density due to the dependence of recombination rate on the carrier energy. However it would be very interesting to carry out similar experiments at constant carrier concentration, with the modulation being caused by the energy exchange between different groups.

8. Conclusions. - During the last decade a better understanding of limitations and advantages of mw technique was gained and modifications of the existing methods were introduced. The mw technique has proved to be a powerful tool for hot electron investigations in various aspects. Especially wide application the $\mathrm{mw}^{\prime} \mathrm{s}$ have found in the study of $v-E$ characteristics of semiconductors exhibiting nam and in investigations of hot electron relaxation effects. Few new trends should be mentioned also: the investigation of breakdown by mw's, hot electron transport in nonuniform structures, the extension of frequency range to submillimeter waves.

In conclusion it should be noted that this review is too brief to do justice to a great deal of excellent work on mot electron studies. The authors apologize for any omission and must confess to the normal subjective view of the subject. 
Acknowledgments. The authors are grateful to A.Matulionis for his critical reading of the manuscript.

\section{References}

/1/ Arthur, G.B., Gibson, A.F., Granville, J.W., J.Flectronics 2 (1956) 145

/2/ Gibson, A.F., Granville,J.W., J.Electronics 2 (1956) 259

13/ Morgan, T.N., Bull.Amer.Phys.Soc. Ser II, 2 (1957)

/4/ Seeger, K., Phys. Rev. 114 (1959) 476

/5/ Vèbra, A., Požela, J., Trudy Akademii Nauk Iit. SSR Ser. B, 2(25) (1961) 99

/6/ Zucker, G., Fowler, V.J., Conwell, E.M., J. Appl. Phys. 32 (1961) 2606

/7/ Inuishi, Y., Hamaguchi, C., J. Phys. Soc. Jpn. 17 (1962) 1813

/8/ Morgan, T.N., Kelly, C.E., Proc. Intern. Conf. Phys. Semicond., Prague (1960), (Publ. Hause Czech. Acad. Sci., 1961) 70

/9/ Gibson, A.F., Granville, J.W., Paige, E.G.S., J.Phys. Chem. Solids 19 (1961) 118

/10/ Brown, M.A.C.S., J.Phys. Chem. Solids 19 (1961) 218

/11/ Banys, r., Požela, J., Lit. Fiz. Sbornik 3 (1963) 419

/12/ Rozenblat, M.A., Doklady Akademii Nauk SSSR 68 (I949) 497

/13/ Carlin, H.J., Pozhela, J.K., Proc. IEEF 53 (1965) 1788

/14/ Schneider, W., Seeger K., Appl. Phys. Lett. 8 (1966) 133

/15/ Hess, K., Seeger, K., Z. Phys. 218 (1969) 431

/16/ Kobayashi, S., Yabuki, S., Aoki, M., Jpn. J. Appl. Phys. 2 (1962) 127

/17/ Seeger, K., J. Appl. Phys. 34 (1962) 1608

/18/ Banys, T., Dargys, A., Pozhela, J., Phys. Stat. Sol. 36 (1969) 755

/19/ Dargys, A., Banys, T., Požela, J., Lit. Fiz. Sbornik 11 (1971) 615

/20/ Dargys, A., Banys, T., Phys. Stat. Sol. 52 (1972) 699

/21/ Kawamura, H., Fukai, M., Hayashi, Y., J. Phys. Soc. Jpn. 17 (I962) 970

/22/ Richter, K., Bonek, E., Phys. Stat. Sol. 31 (1969) 579

/23/ Davydov, A.B., Nomerovany O.M., Prib. Techn. Exper. Nr. 4 (1966) 118

/24/ Evans, A.G.R., Robson, P.N., Sol. St. Electronics 17 (1974) 805 /25/ Bonek, E., J. Appl. Phys. 43 (1972) 5101 
/26/ Heinrich, H., Hess, K., Jantsch, W., Pfeiler, W., J. Phys. Chem. Solids 33 (1972) 425

/27/ Glover, G.H., J. Appl. Phys. 44 (1973) 1295

/28/ Kagan, M.S., Landsberg, E.G., Elenkrig, B.B., Fiz. Techn. Polupr. 10 (1978) 1861

/29/ Dienys, V., Kancleris, ž., Phys. Stat. Sol. (b) 67 (1975) 317

/30/ Hess, K., J. Phys. Chem. Solids 33 (1972) 139

/31/ Dienys, V., Kancleris, ž., Phys. Stat. Sol.(b) 71 (1975) K209

/32/ Dienys, V., Kancleris, ž., Phys. Stat. Sol.(b) 78 (1976) 757

/33/ Sweid, M., Hess, K., Seeger, K., J.Phys. Chem. Solids 39 (1978) 393

/34/ Dienys, V., Martūnas, Z., Fiz. Techn. Polupr. 12 (1978) 1219

/35/ Dienys, V., Kancleris, ž., Martünas, Z., Fiz. Techn. Polupr. I3 (1979) 1706

/36/ Dienys, V., Kancleris, z̆., Miartūnas, Z., Phys. Stat. Sol.(b) IOI (1980) 145

137/ Wind, M., Rapoport, H., Handbook of Microwave Measurements (Polytechn. Inst. Brooklyn, N.Y.) 1965

/38/ Dresselhaus, C., Kip, A.F., Kittel, C., Phys. Rev. 92 (1953) 827

/39/ Rose, K., J. Appl. Phys. 33 (1962) 761

/40/ Borodovsky, P.A., Zimenkov, V.A., Rozentsveig, V.A., Generatsiga SVCh kolebanijs ispolzovaniyem effekta Gunn'a, All-Union Symp., Novosibirsk (1974) (Inst. Sem. Phys., Novosibirsk, 1974) 46

/41/ As̆montas, S.P., Dargys, A.J., Omelyanovski, E.M., Sedrakgan, R. G., Fiz. Techn. Polupr. 9 (1975) 2009

/42/ As̆montas, S.P., Subačius, L.E., Fiz. Techn. Polupr. 13 (1979) 1722

/43/ Ašmontas, S., Dargys, A., Subačius, I., Phys. Stat. Sol.(b) 100 (1980) 691

/44/ Brazis, R., Mironas, A., Filipavičius, A., Fiz. Techn. Polupr. 14 (1980) 1420

/45/ Bruhns, H., Htbner, K., Z. Phys. B 32 (1978) 15

/46/ Curby, R.C., Ferry, D.K., Phys. Stat. Sol.(a) 20 (1973) 569

147/ Dargys, A., Sedrakyan, R., Požela, J., Phys. Stat. Sol.(a) 45 (1978) 387

/48/ Neukermans, A., Kino, G.S., Phys. Rev. B7 (1973) 2703

/49/ Inuishi, Y., Proc. Intern. Symp. on Hicrowave Diagnostics of Semiconductors, Parvoo, Finland (1977) (Helsingsfors, Helsinki 1977), 324 
/50/ Abe, M., Kaneda, S., Jpn. J. Appl. Phys. 11 (1972) 1675

151/ Dargys, A., Žilionis, S., Matulionis, A., Paršeliūnas, J., Požela, J., Poškus, A., Šimulgtè, E., Lit. Fiz. Sbornik 17 (1977) 493

/52/ Pozhela, J.K., Repshas, K.K., Shilalnikas, V.J., Proc. Int. Conf. Phys. Semicond. Exeter (1962), (Inst. Phys, and Phys. Soc., London, 1962) 149

153/ Conwell, E.M., Zucker, J., J. Appl. Phys. 36 (1965) 2192

154/ Ašmontas, S.P., Požela, J.K., Subačius, L.E., Fiz. Techn. Polupr. 11 (1977) 357

155/ Ašmontas, S.P., Olekas, A., Fiz. Techn. Polupr. 14 (1980) 2196

156/ Canali, C., Jacoboni, C., Ottaviani, G., Alberigi-Quaranta, A., Appl. Phys. Lett. 27 (1975)

157/ Imai, I., Kawamura, H., Fukai, M., J. Phys. Soc. Jpn. 2I (1966) 1081

/58/ Gershenson, E.M., Gusinskii, E.N., Phys. Stat. Sol.(b) 64 (1974) 367

159/ Rauch, C.J., Zeiger, H.J., cited in Lax, B., Mavroides, J.G. Solid State Physics 11 (1960) 261 